\title{
Hypoxanthine guanine phosphoribosyltransferase partial deficiency
}

INSERM

\section{Source}

INSERM. (1999). Orphanet: an online rare disease and orphan drug data base. Hypoxanthine guanine phosphoribosyltransferase partial deficiency. ORPHA:79233

Kelley-Seegmiller syndrome (KSS) is the mildest form of hypoxanthine-guanine phosphoribosyltransferase (HPRT) deficiency (see this term), a hereditary disorder of purine metabolism, and is associated with uric acid overproduction (UAO) leading to urolithiasis, and early-onset gout. 